\title{
Hold the Foam: Why Topical Budesonide Remains Relevant for IBD Therapy
}

\author{
Luca Pastorelli ${ }^{1,2} \cdot$ Cristina Bezzio $^{3} \cdot$ Simone Saibeni ${ }^{3}[$
}

Published online: 18 March 2020

(c) Springer Science+Business Media, LLC, part of Springer Nature 2020

The last two decades have witnessed radical changes in the treatment for inflammatory bowel disease (IBD), with an explosion of monoclonal antibody-based 'biologic' drugs targeting components of the immune system. As a consequence, treatment strategies have rapidly evolved, aiming at increasingly ambitious targets.

Despite these major advances, therapy remains far from optimal since achieving and maintaining remission continues to be challenging in many patients. Thus, since IBD treatment can be far from satisfactory for both physicians and patients, it is not surprising that translational and clinical investigation is focused on intestinal inflammation.

Though there is a great interest in developing new drugs and exploiting novel mechanisms to treat IBD, the majority of IBD patients suffer from mild-to-moderately active disease that does not require more aggressive, costly, and potentially harmful therapy. In these cases, significant efforts should also be made in order to improve and maximize the efficacy of already available therapies. For example, molecules with low systemic impact such as 5-aminosalicylates and low-bioavailability topical corticosteroids act directly on inflamed mucosa without achieving significant concentrations in the systemic circulation; these drugs greatly benefit from different administration routes, formulations, and drug delivery systems, which enhance the exposure of therapeutic molecules to the diseased sites.

Moreover, in ulcerative colitis patients needing stronger treatments, topical therapies are important adjunctive treatments since symptoms related to distal inflammation (e.g.,

Simone Saibeni

saibo@tiscali.it

1 Gastroenterology Unit, IRCCS Policlinico San Donato, San Donato Milanese, MI, Italy

2 Department of Biomedical Sciences for Health, University of Milan, Milan, MI, Italy

3 Gastroenterology Unit, Rho Hospital, ASST Rhodense, Corso Europa 250, 20017 Rho, MI, Italy urgency, tenesmus, incontinence, soiling) significantly worsen quality-of-life. Nevertheless, several factors, such as retention problems, unpleasant feeling, rectal/abdominal pain, and flatulence, may limit the adherence to rectal therapies. As a consequence, more comfortable and acceptable topical formulations are welcome in order to further improve adherence with consequent increased efficacy.

Budesonide is a synthetic nonhalogenated glucocorticoid related to triamcinolone hexacetonide, developed in order to reduce systemic side effects from steroids. Oral formulations of budesonide are used in mild-to-moderately active Crohn's disease and ulcerative colitis. Indeed, clinical trials demonstrated the efficacy and safety of topical budesonide formulations in treating active distal forms of IBD, namely ulcerative colitis [1]. Since budesonide foams, it is preferred over enemas by patients [2] and at present is used in several countries.

Although comparative studies historically showed that topical corticosteroids are less effective than topical mesalamine when treating active ulcerative colitis [3], some studies showed similar efficacy between rectal budesonide and rectal 5-ASA [4, 5]. As such, given the theoretical superior anti-inflammatory potency of corticosteroid molecules, it is plausible that there may be room for improvement through optimization of their efficacy. In particular, since effectiveness and tolerability of topical therapy mainly resides in the features of its formulation, attention should focus on identifying different drug release modalities. Indeed, rheological qualities of the rectal formulations strongly influence the time of exposure of the mucosa to the therapeutic molecules, the extent of the colon that can be reached, number of daily administrations, retention time, and patient discomfort.

In this issue of Digestive Diseases and Sciences, Lazaro et al. [6] evaluated the effects of a novel formulation of topical budesonide in a chemically induced mice model of colitis. In detail, they used an inclusion complex of budesonide/hydroxypropyl-beta-cyclodextrin, a formulation that increases drug solubility, incorporated in poloxamer 
hydrogels; interestingly, the suspension resulting from the incorporation of cyclodextrin into poloxamer-based gels possesses the capacity to change its physical state and qualities in response to minor temperature variations. At room temperature, the suspension is a liquid, and, thus, when administered as enema, can easily ascend proximally and reach the splenic flexure. On the other hand, at physiological body temperature, the mixture becomes semisolid, thus easier to retain, persistently adhering to the colonic mucosa, enhancing exposure time to the therapeutic compounds. Furthermore, the formation of drug-containing micelles leads to micelle-micelle interactions that support persistent drug release. The authors analyzed weight loss, tissue myeloperoxidase activity, macroscopic and microscopic inflammatory activity, pro-inflammatory cytokine expression (TNF and interleukin-1 $\beta$ ), and inhibition of endogenous steroid production in a rat IBD model. Results demonstrated that the incorporation of budesonide into poloxamer hydrogels was superior to plain or hydroxypropyl-beta-cyclodextrinincluded budesonide in the reduction of intestinal inflammation; indeed, the administration of poloxamer hydrogels alone, without budesonide, quite surprisingly showed a trend toward a better efficacy than the control budesonide formulations, suggesting that poloxamer hydrogels might possess anti-inflammatory properties in and of themselves.

In recent years, several new different budesonide topical formulations have been proposed in preclinical studies conducted in animal models [7, 8]. In particular, a formulation with similar rheological properties to the compound studied by Lazaro et al. (thermosensitive drug delivery platform, based on hydrophilic polyethylene glycol hydrophobic polypropylene glycol blocks) has been described and tested in murine dextran sodium sulfate chemically induced colitis; likewise, this formulation proved to be superior to liquid budesonide in controlling colonic inflammation [8]. Still, the interest and novelty of the data presented in this issue of Digestive Diseases and Sciences remain very high; in fact, this approach, if translated to IBD patients, holds the potential to significantly enhance the effectiveness of topical therapies and may theoretically be applied to other topical drugs, not only mesalamine and beclomethasone dipropionate, but also inhibitors of tumor necrosis factor-alpha or other cytokines, as well. Still, although results have to be confirmed in humans by means of phase 1 clinical studies, translation to IBD patients may not be as linear as expected; indeed, chemically induced models of colitis may not fully recapitulate human disease, due to the marked differences between all experimental IBD models and human disease. Moreover, factors such as discomfort in auto-administering rectal therapies and compliance and adherence, together with the extent of colon reached by the enema/foam, which are pivotal elements in managing human disease, cannot be accurately predicted in preclinical studies conducted on animal models. Nonetheless, Lazaro's manuscript strongly paves the way to future clinical studies.

The discovery and introduction in clinical practice of novel therapeutic agents have always marked exciting moments in the history of IBD therapy. Despite vast resources spent by pharmaceutical companies in order to launch new drugs with innovative mechanisms of action, in the era preceding the introduction of biologic IBD therapies, substantial progress was made by improving existing drug formulations. Perfecting drug formulations enables the more efficient targeting the anatomical site of the disease, prolongation of drug exposure time, and the reduction in the amounts of therapeutic molecules reaching nontarget systems or organs, thus reducing adverse effects. Since this type of therapeutic optimization remains of utmost importance, it is reassuring that this type of work remains important for researchers and pharmaceutical companies.

Since IBD is a lifelong disease, any drug may lose its efficacy over time. While looking for new, potent, and groundbreaking weapons in the therapeutic arsenal, clinicians should not lose sight of proven therapies, constantly endeavoring to find innovative ways to employ them.

\section{Compliance with Ethical Standards}

Conflict of interest The authors declare that they have no conflict of interest.

\section{References}

1. Zeng J, Lv L, Mei ZC. Budesonide foam for mild to moderate distal ulcerative colitis: a systematic review and meta-analysis. J Gastroenterol Hepatol. 2017;32:558-566.

2. Gross V, Bar-Meir S, Lavy A, International Budesonide Foam Study Group, et al. Budesonide foam versus budesonide enema in active ulcerative proctitis and proctosigmoiditis. Aliment Pharmacol Ther. 2006;2:303-312.

3. Marshall JK, Thabane M, Steinhart AH, et al. Rectal 5-aminosalicylic acid for induction of remission in ulcerative colitis. Cochrane Database Syst Rev. 2010. https://doi. org/10.1002/14651858.CD004115.pub2.

4. Lémann M, Galian A, Rutgeerts P, et al. Comparison of budesonide and 5-aminosalicylic acid enemas in active distal ulcerative colitis. Aliment Pharmacol Ther. 1995;9:557-562.

5. Kruis W, Neshta V, Pesegova M, et al. Budesonide suppositories are effective and safe for treating acute ulcerative proctitis. Clin Gastroenterol Hepatol. 2019;17:98-106.

6. Lázaro CM, de Oliveira CC, Gambero A, et al. Evaluation of Budesonide-Hydroxypropyl- $\beta$-Cyclodextrin inclusion complex in thermoreversible gels for ulcerative colitis. Dig Dis Sci. 
(Epub ahead of print). https://doi.org/10.1007/s10620-02006075-y.

7. Date AA, Halpert G, Babu T, et al. Mucus-penetrating budesonide nanosuspension enema for local treatment of inflammatory bowel disease. Biomaterials. 2018;185:97-105.

8. Sinha SR, Nguyen LP, Inayathullah M, et al. A thermo-sensitive delivery platform for topical administration of inflammatory bowel disease therapies. Gastroenterology. 2015;149:52-55.e2.
Publisher's Note Springer Nature remains neutral with regard to jurisdictional claims in published maps and institutional affiliations. 